\title{
NOTE ON A PAPER OF E.M.E. ZAYED AND S.F.M. IBRAHIM
}

\author{
W. EBERHARD, G. FREILING
}

and

\section{A. SCHNEIDER}

(Received September 4, 1991 and in revised form December 20, 1991)

In [1] E.M.E. Zayed and S.F.M. Ibrahim consider the fourth order eigenvalue problem

$$
\begin{array}{ll}
\left(K u^{\prime \prime}\right)^{\prime \prime}-\left(P u^{\prime}\right)^{\prime}+q u & =\lambda u, \quad x \in[a, b] \\
u(a)=\left(P u^{\prime}\right)(a) & =\left(K u^{\prime \prime}\right)(a)=0 \\
\left(K u^{\prime \prime \prime}\right)(b)-\left(P u^{\prime}\right)(b) & =-\lambda u(b),
\end{array}
$$

,where $K, P$ and $q$ are assumed to be continuous real valued functions on $[a, b]$ with $K(x)>0, P(x)>0, q(x)>0$. They introduce a linear operator $A$ in the Hilbert space $H=L^{2}([a, b]) \times C$ such that $\lambda$ is an eigenvalue of (1) and $u$ a corresponding eigenfunction if and only if $\lambda$ is an eigenvalue of $A$ and $(u, u(b))$ a corresponding eigenvector. Then the authors assert (see Remark 3.2) that $A$ has a sequence of positive eigenvalues $\lambda_{i}$ with $\lambda_{i} \rightarrow+\infty$ as $i \rightarrow+\infty$ and that the set of the orthonormalized eigenvectors form a complete orthonormal system in $H$.

Since problems of the form (1.1) occur in connection with technical problems (e.g. the description of motions of helicopter blades) we want to point out, that the assertions of the paper [1] cannot be true, since they contradict well-known classical results. We demonstrate this by considering the case $K(x) \equiv 1$, where the problem (1) reduces to

$$
\begin{aligned}
& y^{(4)}-\left(P y^{\prime}\right)^{\prime}+q y=\lambda y \\
& y(a)=\left(P y^{\prime}\right)(a)=y^{\prime \prime}(a)=0 \\
& y^{\prime \prime \prime}(b)-\left(P y^{\prime}\right)(b)=-\lambda y(b) .
\end{aligned}
$$

This is a problem with separated boundary conditions (see [2]). In the case of $\lambda$ independent boundary conditions formula $(28 \mathrm{~b})$ p. 94 in [2] shows, that there exists a sequence of eigenvalues with the only accumulation point $-\infty$, since we have three conditions in $a$ and one condition in $b$. It can easily be verified, that the same result remains valid, if we have the $\lambda$-dependent boundary condition of the given form at $b$. For the proof we set $\lambda=\rho^{4}, S_{0}=\left\{\rho \mid 0 \leq \arg \rho \leq \frac{\pi}{2}\right\}$ and consider for $(x, \rho) \in[a, b] \times S_{0}$ the asymptotic estimates ([2], p. 49)

$$
y_{i}^{(k)}=\left(\rho \omega_{i}\right)^{k} e^{\rho \omega_{1}(x-a)}\left[1+O\left(\frac{1}{\rho}\right)\right], \quad i=1, \ldots, 4 ; \quad k=0,1,2,3
$$

for a suitable fundamental system $y_{i}=y_{i}(x, \rho)$ of the differential equation in (2) where $\omega_{1}=-1, \omega_{2}=i, \omega_{3}=-i, \omega_{4}=1$.

By plugging (3) into the boundary-expressions we obtain 


$$
\begin{aligned}
& y_{i}^{(k)}(0)=\left(\rho \omega_{i}\right)^{k}\left[1+O\left(\frac{1}{\rho}\right)\right], \quad k=0,1,2 \\
& \left.y_{i}^{\prime \prime \prime}(b)-P(b) y_{i}^{\prime}(b)+\lambda y_{i}(b)=\left(\rho^{4}-P(b) \rho \omega_{i}+\left(\rho \omega_{i}\right)^{3}\right) e^{\rho \omega_{1}(b-a)}\right)\left[1+O\left(\frac{1}{\rho}\right)\right] \\
& =\rho^{4} e^{\rho \omega_{i}(b-a)}\left[1+O\left(\frac{1}{\rho}\right)\right]
\end{aligned}
$$

Therefore the characteristic determinant of (2) can be estimated asymptotically for $\rho \in S_{0}$ by

$$
\begin{gathered}
\Delta(\rho)=P(a) \rho^{7}\left|\begin{array}{cccc}
e^{\rho \omega_{1}(b-a)} & e^{\rho \omega_{2}(b-a)} & e^{\rho \omega_{3}(b-a)} & e^{\rho \omega_{4}(b-a)} \\
1 & 1 & 1 & 1 \\
\omega_{1} & \omega_{2} & \omega_{3} & \omega_{4} \\
\omega_{1}^{2} & \omega_{2}^{2} & \omega_{3}^{2} & \omega_{4}^{2}
\end{array}\right|\left[1+O\left(\frac{1}{\rho}\right)\right] \\
=\rho^{7} \sum_{i=1}^{4} c_{i} e^{\rho \omega_{1}(b-a)}\left[1+O\left(\frac{1}{\rho}\right)\right] \text { with } c_{i} \neq 0 .
\end{gathered}
$$

To the representation (5) of $\Delta$ we can apply the same considerations as in the proof of (28b) in [2], p. 94, obtaining the asymptotic distribution of the eigenvalues $\lambda_{k}=\rho_{k}^{4}$ of (2) by the simplified formula

$$
\rho_{k}=\frac{\sqrt{2} \pi}{b-a} e^{\frac{2 \pi}{4}} k\left[1+O\left(\frac{1}{k}\right)\right] \quad k \in N,
$$

implying the assertion that $-\infty$ is the only accumulation point of the sequence $\left\{\lambda_{k}\right\}$. (This formula is a special case of Lemma 1 in [3] a), p. 265.)

Hence we can state:

Lemma 1.3: The eigenvalue problem (2) has a countable set of eigenvalues with the only accumulation point $-\infty$. Almost all eigenvalues have multiplicity 1 .

There is a second point to be clearified. Zayed and Ibrahim have defined the operator $A$, whose spectrum is identical with the set of eigenvalues of (1), but for their discussion they make the additional assumption (5) p. 343:

$$
K^{\prime}(b) u(b)-K(b) u^{\prime}(b)=0 .
$$

Consequently they consider the restriction $B$ of $A$ to the subspace $D(B):=\{(y, y(b)) \in$ $\left.D(A) \mid K^{\prime}(b) y(b)-K(b) y^{\prime}(b)=0\right\}$. With respect to our example (2) this restriction $B$ describes the eigenvalue problem

$$
\begin{aligned}
& y^{(4)}-\left(P y^{\prime}\right)^{\prime}+q y=\lambda y \\
& y(a)=P y^{\prime}(a)=y^{\prime \prime}(a)=0 \\
& y^{\prime \prime \prime}(b)-\left(P y^{\prime}\right)(b)=-\lambda y(b) \\
& y^{\prime}(b)=0 .
\end{aligned}
$$

Obviously every eigenvalue of (2') is also an eigenvalue of (2) and thus $-\infty$ is the only possible accumulation point. On the other hand it is easily verified, that for every eigenvalue $\lambda$ and for the corresponding eigenfunction $y$ of (2') we get the equation

$$
\lambda\left\{\int_{0}^{b}|y(x)|^{2} d x+|y(b)|^{2}\right\}=\int_{0}^{b}\left(\left|y^{\prime \prime}(x)\right|^{2}+P(x)\left|y^{\prime}(x)\right|^{2}+q(x)|y(x)|^{2}\right) d x,
$$

so that (2') may have only positive eigenvalues. Hence (2') has at most a finite set of eigenvalues (of finite multiplicity) and therefore the system of eigenvectors of the operator $B$ never can form a complete orthonormal set in $H$, since $\operatorname{dim} H=+\infty$. 
We note that (1) is a normal but irregular (not almost regular) eigenvalue problem (in the sense of [4]). Concerning the expansion of arbitrary functions into a uniformly convergent series in terms of eigenfunctions of irregular eigenvalue problems we refer to the works of Ward, Khromov or Eberhard, cited in [2] and of Freiling [3] where it is shown that uniformly convergent expansions in terms of eigenfunctions can only be obtained for a very small class of infinitely differentiable functions. Additional results on irregular problems with separated boundary conditions can be found in the papers cited in [3].

\section{References}

[1 ] Zayed, E.M.E. and Ibrahim, S.F.M.: Eigenfunction expansion for a regular fourth order eigenvalue problem with eigenvalue parameter in the boundary conditions. Internat. J. Math. and Math. Sci., Vol. 12 No 2 (1989), 341-348.

[2 ] Naimark, M.A.: Linear Differential Operators, Part I, F. Ungar, New York (1967).

.[3 ] Freiling, G.:

a) Zur Vollständigkeit des Systems der Eigenfunktionen irregulärer Eigenwertprobleme mit $\lambda$-abhängigen Randbedingungen. Zeitsch. f. Anal. u. Anw. 3 (1984), 263-269.

b) Necessary conditions for the $L_{2}$-convergence of series in eigenfunctions of irregular eigenvalue problems. J. Math. Anal. Appl. 114 (1986), 503-511.

c) Necessary conditions for the uniform convergence and Abel-summability of eigenfunction expansions with irregular ordinary differential bundles. Zeitschrift Analysis Anw. 5 (1986), 543-552.

[4 ] Shkalikov, A.A.: Boundary value problems for ordinary differential equations with a parameter in the boundary conditions. Journal of Soviet Math. 33, 1311-1342 (1986), Transl. of: Trudy Sem. imeni Petrovskogo 9 (1983), 190-229 . 


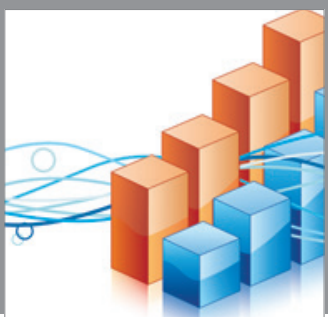

Advances in

Operations Research

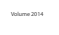

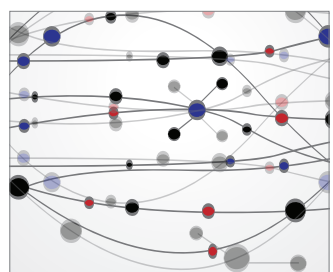

\section{The Scientific} World Journal
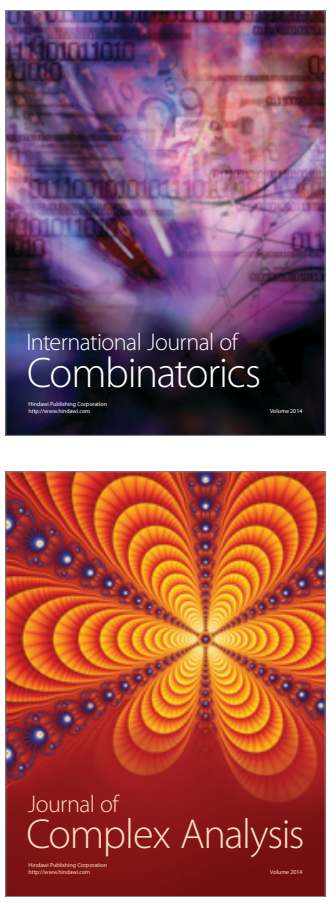

International Journal of

Mathematics and

Mathematical

Sciences
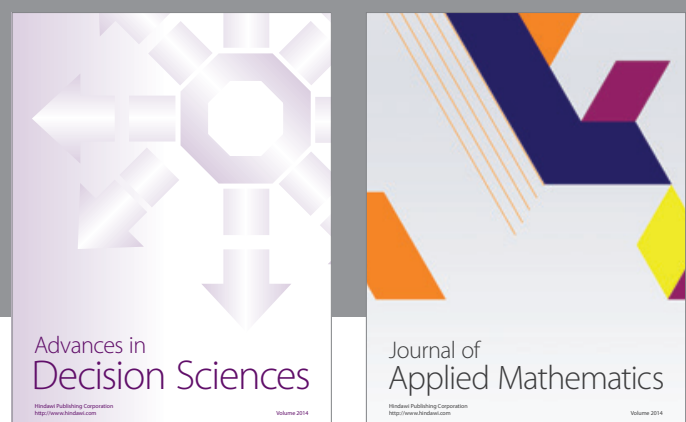

Journal of

Applied Mathematics
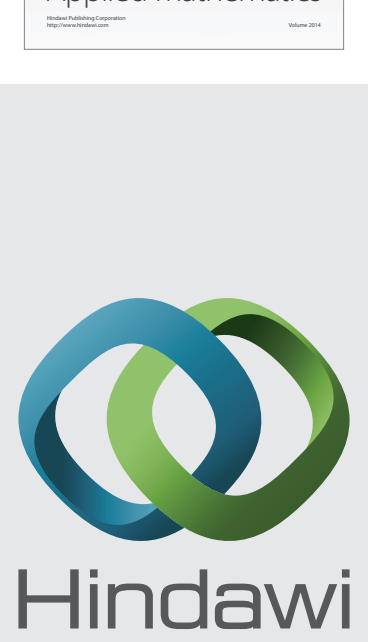

Submit your manuscripts at http://www.hindawi.com
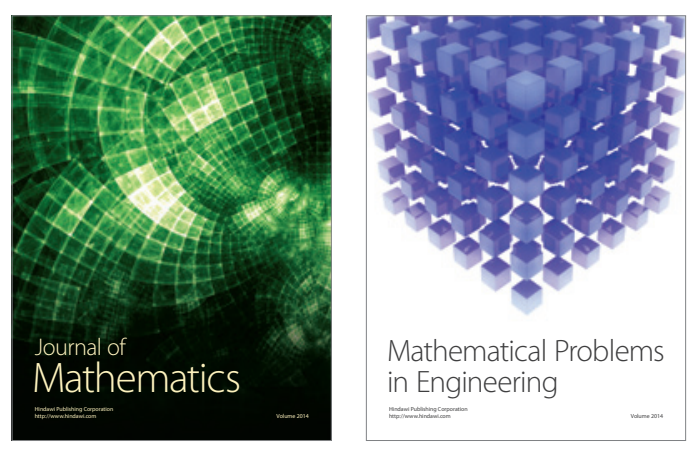

Mathematical Problems in Engineering
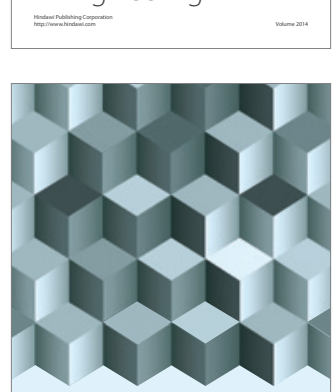

Journal of

Function Spaces
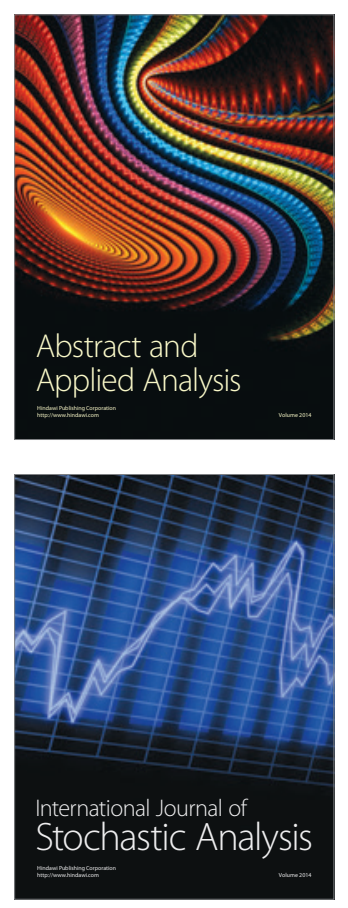

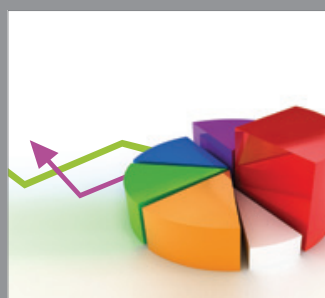

ournal of

Probability and Statistics

Promensencen
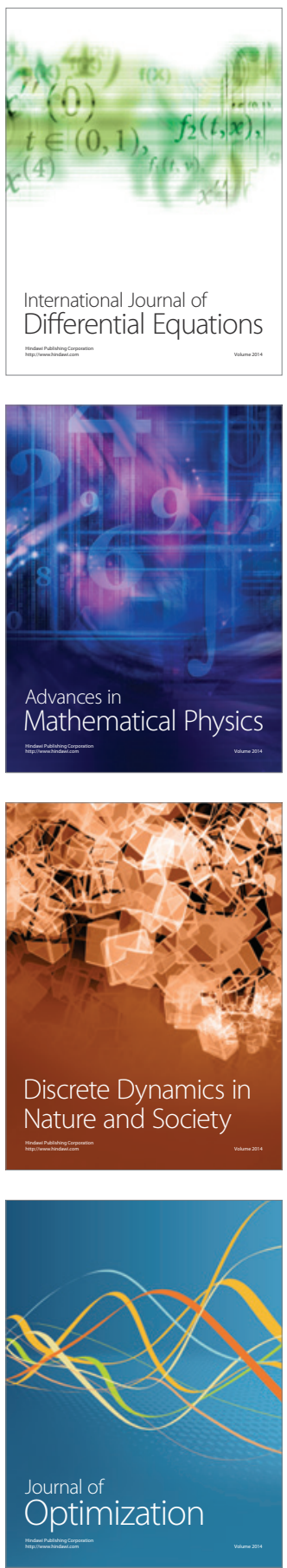